\title{
Erratum: "Effect of Electric Field on NMR Spectra in Centroantisymmetric Antiferromagnets" [Phys. Solid State 42 (5), 903 (2000)]
}

\author{
V. V. Leskovets and E. A. Turov
}

On page 906, Eq. (17) should have the form

$$
\begin{gathered}
\Omega_{1,4}^{2}=A L_{y^{\prime}}\left[\left(A L_{y^{\prime}}-2 M_{y^{\prime}}\right)+2\left(-\lambda_{123} \pm \lambda_{113}\right) L_{y^{\prime}} E_{z}\right], \\
\Omega_{2,3}^{2}=A L_{y^{\prime}}\left[\left(A L_{y^{\prime}}+2 M_{y^{\prime}}\right)+2\left(\lambda_{123} \pm \lambda_{113}\right) L_{y^{\prime}} E_{z}\right] .
\end{gathered}
$$

In the next paragraph, $\lambda_{123}$ should be replaced by $\lambda_{113}$. 